\title{
SI USTED FUERA MIGRANTE. LAS PREFERENCIAS DE CANDIDATO COMO HIPÓTESIS ${ }^{1}$
}

\author{
IF YOU WERE A MIGRANT. CANDIDATE PREFERENCE AS A \\ HYPOTHESIS
}

\author{
Antonio Alaminos Chica \\ Universidad de Alicante (Spain) \\ alaminos@ua.es \\ Clemente Penalva Verdú \\ Universidad de Alicante (Spain) \\ clemente.penalva@ua.es \\ Ignacia Perea Crespo \\ OBETS - Universitat d'Alacant (Spain) \\ nachicrespo@gmail.com
}

\section{Resumen}

A partir de los datos de una encuesta sobre el conjunto de países de la Unión Europea (UE25), se intenta acceder a la manera en que las sociedades europeas asocian sus concepciones sobre ciudadanía (a partir de la posible traslación del derecho al sufragio en casos de movilidad geográfica) con sus preferencias electorales (en torno a la dicotomía del voto al candidato del país de destino o al candidato de su propio país) en el caso hipotético de que los encuestados migraran a otro país de la Unión Europea. Los resultados muestran una asociación significativa entre cuatro tipos de orientaciones sobre ciudadanía en

\footnotetext{
${ }^{1}$ Este estudio ha sido financiado por el proyecto CSO2012-32930 “La participación política como candidatos de los residentes europeos en España", Ministerio de Economía y competitividad. Los datos proceden de Flash Barómetro 364.
} 
casos de movilidad (orientada a la nación, orientada al Estado, cosmopolita y pérdida de derechos) y el origen del candidato al que se dirigen las preferencias de voto. Las orientaciones nacionales se asocian a una preferencia sobre candidatos connacionales, mientras que las orientaciones estatales se asocian a una preferencia sobre candidatos del país de destino. Las conclusiones de este estudio exploratorio permite una reflexión sobre los factores explicativos que inciden sobre esta estructura de opiniones sobre la nacionalidad y la ciudadanía (observando el contexto actual de crisis económica y social); y sobre el efecto que esta configuración de las opiniones públicas puede tener sobre el futuro de la Unión Europea como proyecto político supranacional y democrático.

Palabras clave: ciudadanía, nacionalidad, movilidad intraeuropea, Unión Europea, Opinión Pública

\section{INTRODUCCIÓN}

Este estudio aborda las preferencias electorales de los ciudadanos europeos en un contexto de movilidad interestatal, reconocido y promovido por la Unión Europea. Considerando la intensidad de la movilidad de los últimos años en el espacio europeo, la configuración de las sociedades políticas de los diferentes estados miembros ha cambiado, reconociéndose la posibilidad real y teórica de unas sociedades políticas plurinacionales donde se incorporan de forma progresiva ciudadanos europeos que votan, crean y se presentan como candidatos de organizaciones políticas nuevas, formadas, fundamentalmente a nivel local, por ciudadanos cuyo origen es de un país diferente de aquel en el cual residen. La novedad política que provoca mayor disonancia para los electores autóctonos es encontrar partidos nuevos formados por residentes extranjeros, pero también encontrar listas de partidos, llamemos tradicionales, salpicados por candidatos con nombres extranjeros. Desde este presupuesto, el de asistir a una situación política nueva, la intención de este trabajo es estudiar hasta qué punto las preferencias electorales de los ciudadanos europeos se guían por la presencia de candidatos connacionales o no; o si es indiferente la nacionalidad de los candidatos como criterio para decidir un voto.

Aunque el voto no es la única forma de participación política, se parte de la idea de que el voto condensa gran parte de los derechos adquiridos por la población migrante en un país de destino. La capacidad de constituirse como candidatos o como electores, tiene una utilidad práctica (articular la representación de los propios intereses o ideología) y simbólica (reconocimiento directo de los derechos políticos y voluntad de integración en el sistema político nacional). Aunque son escasos, se conocen los estudios sobre las actitudes y comportamiento político de los migrantes. Lo que no se conoce es la predisposición al voto de las personas que no son emigrantes, pero que lo son 
potencialmente. Esta es la novedad de este estudio. Aprovecha la presencia en el Eurobarómetro de 2012 de unas preguntas que incitan al encuestado a ubicarse en un contexto social y político nuevo para a continuación preguntar sobre cuáles serían sus preferencias políticas en esa situación hipotética. No es lo mismo pasar por la experiencia de la migración internacional que no haberla experimentado, pero el ejercicio proyectivo de situarse hipotéticamente en esta tesitura de movilidad puede dar pistas sobre qué es lo que se piensa acerca de los límites de los derechos políticos y sociales de los migrantes con los que se convive $\mathrm{y}$, también de forma indirecta, inferir el grado de consistencia de sus predisposiciones al voto y su concepción de ciudadanía en un contexto como el actual, marcado por la movilidad internacional.

El estudio de las preferencias políticas de los migrantes y su comportamiento electoral, $y$, en especial desde un punto de vista comparativo, constituye un desafío de investigación importante, entre otras razones por la escasez de datos. Son varios los motivos detrás de estas limitaciones, como son la diversidad de países de origen en las corrientes migratorias, la fragmentación de los asentamientos, su elevada movilidad geográfica en los destinos, la diversidad lingüística o la posición social, que con frecuencia es marginal. Todos estos factores interaccionan produciendo una heterogeneidad tan elevada que el concepto mismo de migrante presenta contenidos socioeconómicos muy diversos.

Bird, Saalfeld y Wüst (2011) en su estudio sobre el comportamiento electoral de los inmigrantes indican la dificultad para obtener datos comparados.

The main conclusions are that suitable data are scarce and analyses are often quite idiosyncratic. Data source range from voter registers (Scandinavia), partially cumulated general surveys (eg. Canada. UK. Germany). to special minority-focused surveys (e g. France. Netherlands, Austria. UK). In none of the countries are data available for all political levels. While some countries focus largely or exclusively on local elections, others look almost exclusively at the national level. This difference in the scale of analysis is clearly linked to state-level variations in voter eligibility. The chapter also shows that there are hardly any common standards for identifying eligible voters with an immigrant or visible minority background (exceptions are Canada and the UK). Consequently, the conditions for comparative analyses across countries are modest.

A la diversidad de las poblaciones etiquetadas como migrantes, y en especial sus diferentes culturas políticas de origen, se suma la diversidad de modelos alternativos que se ofrecen como explicativos del comportamiento electoral: características sociodemográficas de la Escuela de Columbia; adhesiones individuales de largo plazo sobre determinados grupos sociales, y el rol de 
temáticas y candidatos de la Escuela de Michigan; y los cálculos económicos en términos de coste y beneficios y la proximidad ideológica para la Escuela de Elección Racional. En esta investigación, el enfoque que se emplea es de nivel macro, de tal modo que siendo como unidad de análisis el Estado, las diferencias de carácter individual (género, edad, status socioeconómico, posicionamientos sobre diferentes propuestas políticas o ideológicas) quedan resumidas en categorías amplias.

En el caso europeo que nos ocupa, el marco legal de participación electoral en elecciones locales y europeas, en lo que se refiere a los migrantes procedentes de otros países de la Unión Europea, se encuentra regulado de forma estándar. Así, un ciudadano de la Unión Europea que desee ejercer el derecho al sufragio tanto activo como pasivo en las elecciones locales o europeas, puede hacerlo en las condiciones de registro que especifique cada país. El poder explicativo de la teoría de la Estructura de Oportunidades Políticas (Tarrow, 1998) para la participación de los emigrantes se atenúa, al menos en lo referido a las facilidades legales, si bien permanece válido para otros aspectos políticos, económicos o sociales.

El objetivo central de este estudio considera la preferencia que un votante puede mostrar, en el caso hipotético de migrar a otro país, por candidatos del país de origen o del país de residencia. Para ello, emplea una propuesta bastante interesante desde el punto de vista metodológico. Dado que estudiar las preferencias electorales de los migrantes europeos en sus países de residencia es de un coste elevado, y que los datos disponibles son realmente fragmentarios y de fiabilidad limitada, se aprovecha el planteamiento de una pregunta dirigida a la población general con un doble condicional. Así, en una encuesta europea estándar, "Flash Eurobarometer" 364 (European Commission, Brussels, 2012), a la población general, se encadena la siguiente secuencia en una pregunta: a) imagine que usted ha emigrado a otro país europeo, y b) imagine que para las listas de las elecciones europeas usted puede elegir entre candidatos de su país de origen o candidatos del país europeo donde tiene su residencia. En esas condiciones, ¿Qué candidato prefiere, uno de su país de origen o uno de su país de residencia?

Es evidente que cabe inferir, con todas sus dificultades, que dichas preferencias pueden extrapolarse al caso real de emigrar. Entre las dificultase se encuentran, de forma evidente, el hecho de que los que realmente emigran puedan ser unas subpoblaciones poco representativas en términos de heterogeneidad respecto a la población general. Pero, en el planteamiento positivo, como ventaja, el recurso a las elecciones europeas controla la naturaleza específica que poseen las elecciones locales, en las que establecer una hipótesis de candidatos es realmente poco realista. Las elecciones europeas, por su referencia trasversal facilita la comparación entre los tipos de candidatos. 
En todo caso, cabe recordar que con la hipótesis de migración la pregunta adquiere unas propiedades proyectivas que facilita una respuesta más libre y sincera. En términos contrarios, como desventaja, las preguntas que consideran hipótesis tienen como consecuencia, por lo general, un porcentaje elevado de no respuesta (Alaminos, 1994). Al menos en poblaciones o subpoblaciones con baja capacidad especulativa.

En segundo lugar, tras la hipótesis de migración, las preferencias de candidato son evaluadas en función de la orientación sobre los derechos políticos en Europa. Así, en un artículo reciente (Alaminos, Penalva y Perea, 2017) Se determina una tipología de electores en función a su opinión sobre hasta dónde ha de llegar la adquisición o pérdida del derecho al sufragio activo en elecciones nacionales, explorando su relación con la propuesta de clúster de culturas cívico-políticas en la Unión Europea. Asimismo, se analiza su dimensionalidad exponiendo dos dimensiones principales, la primera identifica el eje nación/Estado y la segunda el eje sin-derechos/cosmopolita. Sobre el plano formado por ambas dimensiones, los países se sitúan en cuatro categorías.

Tabla 1. Concepción de la ciudadanía en función del derecho de sufragio en las elecciones generales tras la migración.

\begin{tabular}{|c|c|c|c|}
\hline & \multicolumn{2}{|c|}{ Derecho de sufragio activo en país de residencia } \\
\hline & & No & Sí \\
\hline \multirow{2}{*}{$\begin{array}{l}\text { Derecho de sufragio } \\
\text { activo en país de origen }\end{array}$} & No & Sin-derechos orientada & $\begin{array}{c}\text { Ciudadanía } \\
\text { aristotélica } \\
\text { (Estado orientada) }\end{array}$ \\
\hline & Sí & $\begin{array}{l}\text { Ciudadanía Romana } \\
\text { (nación orientada) }\end{array}$ & $\begin{array}{l}\text { Ciudadanía } \\
\text { cosmopolita }\end{array}$ \\
\hline
\end{tabular}

Source: Alaminos, Penalva, Perea (2017)

En esta tipología propuesta se pueden observar la proporción para cada país de individuos que están dentro de las siguientes categorías sobre la concepción del derecho a la participación política electoral en caso de movilidad intraeuropea. Estas cuatro formas de concebir el derecho a voto provienen del cruce de las respuestas a dos preguntas.

"P2.- Consideremos una situación en la que un ciudadano de la UE vive en otro estado miembro distinto del suyo de origen (es decir, de donde tenga la ciudadanía):

(1) ¿Debería este ciudadano perder su derecho a votar en unas elecciones nacionales en el país de donde tenga la ciudadanía?

(2) ¿Debería este ciudadano tener derecho a votar y a presentarse como candidato en unas elecciones nacionales organizadas en el estado miembro donde reside?" 
La tipología establecida establece, por tanto, estas cuatro concepciones de la ciudadanía:

A. Ciudadanía sin derechos-orientad. No vota en origen y no vota en destino: el individuo pierde el derecho al voto cuando viaja. Se pierde el derecho al sufragio con el desarraigo.

B. Ciudadanía Estado-orientada. El ciudadano mantiene el derecho al voto en su estado de origen y no lo adquiere en el de destino. Derecho anclado en el estado de origen.

C. Ciudadanía nación-orientada. El ciudadano migrante adquiere el derecho al voto allí donde va, pero pierde el de origen. Es un derecho anclado al espacio del Estado de destino.

D. La orientación cosmopolita reconoce el derecho a votar en elecciones generales tanto en su país de origen y como en su país de destino. En definitiva, el individuo elige en qué país votar, sin exclusiones.

Los resultados obtenidos para cada categoría en cada Estado de la Unión ofrece la configuración que aparece en la tabla 2.

\section{DATOS E HIPÓTESIS}

Los datos empleados para la prueba de hipótesis proceden del estudio de opinión pública de la Comisión Europea (European Commission, Brussels, 2012). El planteamiento de la pregunta intenta colocar psicológicamente al entrevistado como residente en un país europeo diferente al propio e interroga sobre cómo sería su decisión de voto a) sobre candidatos de su propio país (se entiende que coincide el sufragio activo y el pasivo sobre la misma nacionalidad de origen) o b) sobre candidatos dispares en términos nacionales del sufragio activo (residente extranjero) y el pasivo (candidato del país de destino). Así está planteada la pregunta en el cuestionario:

Q3 If you are living or were to live in another EU country than your country of origin (i.e. of which you are a national) would you rather vote in European elections for "the candidates of your country of residence or the candidates of your country of origin". 
Si usted fuera migrante. Las preferencias de candidato como hipótesis

Tabla 2. Tipología de ciudadanía según país europeo (2012)

\begin{tabular}{|l|r|r|r|r|r|r|}
\hline & $\begin{array}{c}\text { No } \\
\text { rights- } \\
\text { oriented }\end{array}$ & $\begin{array}{c}\text { Nation- } \\
\text { oriented }\end{array}$ & $\begin{array}{c}\text { State- } \\
\text { oriented }\end{array}$ & $\begin{array}{c}\text { Cosmopolitanist- } \\
\text { oriented }\end{array}$ & No sabe & Total \\
\hline Austria & $9,50 \%$ & $27,10 \%$ & $21,40 \%$ & $34,90 \%$ & $7,20 \%$ & $100 \%$ \\
\hline Belgium & $10,00 \%$ & $19,30 \%$ & $27,90 \%$ & $36,60 \%$ & $6,30 \%$ & $100 \%$ \\
\hline Bulgaria & $6,50 \%$ & $21,60 \%$ & $17,50 \%$ & $47,70 \%$ & $6,60 \%$ & $100 \%$ \\
\hline Cyprus (Republic) & $7,90 \%$ & $24,10 \%$ & $18,90 \%$ & $43,80 \%$ & $5,30 \%$ & $100 \%$ \\
\hline Czech Republic & $7,60 \%$ & $25,40 \%$ & $17,30 \%$ & $42,20 \%$ & $7,60 \%$ & $100 \%$ \\
\hline Germany & $5,80 \%$ & $20,40 \%$ & $24,40 \%$ & $43,10 \%$ & $6,40 \%$ & $100 \%$ \\
\hline Denmark & $8,20 \%$ & $38,20 \%$ & $22,50 \%$ & $24,60 \%$ & $6,50 \%$ & $100 \%$ \\
\hline Estonia & $8,90 \%$ & $25,10 \%$ & $12,60 \%$ & $34,70 \%$ & $18,70 \%$ & $100 \%$ \\
\hline Spain & $6,80 \%$ & $23,20 \%$ & $19,60 \%$ & $44,30 \%$ & $6,10 \%$ & $100 \%$ \\
\hline Finland & $7,70 \%$ & $29,40 \%$ & $13,30 \%$ & $41,50 \%$ & $8,10 \%$ & $100 \%$ \\
\hline France & $9,50 \%$ & $29,60 \%$ & $18,00 \%$ & $40,20 \%$ & $2,80 \%$ & $100 \%$ \\
\hline United Kingdom & $11,70 \%$ & $14,20 \%$ & $32,60 \%$ & $33,70 \%$ & $7,90 \%$ & $100 \%$ \\
\hline Greece & $6,10 \%$ & $21,30 \%$ & $17,80 \%$ & $48,30 \%$ & $6,40 \%$ & $100 \%$ \\
\hline Hungary & $7,60 \%$ & $32,00 \%$ & $14,10 \%$ & $38,50 \%$ & $7,90 \%$ & $100 \%$ \\
\hline Ireland & $6,40 \%$ & $9,40 \%$ & $31,70 \%$ & $47,70 \%$ & $4,90 \%$ & $100 \%$ \\
\hline Italy & $5,50 \%$ & $17,20 \%$ & $25,00 \%$ & $44,30 \%$ & $8,00 \%$ & $100 \%$ \\
\hline Lithuania & $9,60 \%$ & $31,50 \%$ & $15,90 \%$ & $34,40 \%$ & $8,60 \%$ & $100 \%$ \\
\hline Luxembourg & $9,30 \%$ & $17,80 \%$ & $27,30 \%$ & $41,50 \%$ & $4,20 \%$ & $100 \%$ \\
\hline Latvia & $11,80 \%$ & $30,40 \%$ & $15,90 \%$ & $33,90 \%$ & $8,10 \%$ & $100 \%$ \\
\hline Malta & $7,70 \%$ & $18,10 \%$ & $29,30 \%$ & $37,70 \%$ & $7,30 \%$ & $100 \%$ \\
\hline The Netherlands & $10,30 \%$ & $25,10 \%$ & $23,00 \%$ & $35,90 \%$ & $5,80 \%$ & $100 \%$ \\
\hline Poland & $6,50 \%$ & $21,60 \%$ & $18,10 \%$ & $50,20 \%$ & $3,60 \%$ & $100 \%$ \\
\hline Portugal & $4,70 \%$ & $19,00 \%$ & $18,20 \%$ & $55,50 \%$ & $2,60 \%$ & $100 \%$ \\
\hline Romania & $6,90 \%$ & $13,90 \%$ & $21,70 \%$ & $52,20 \%$ & $5,30 \%$ & $100 \%$ \\
\hline Sweden & $6,40 \%$ & $25,80 \%$ & $17,50 \%$ & $42,60 \%$ & $6,20 \%$ & $100 \%$ \\
\hline Slovenia & $12,00 \%$ & $22,40 \%$ & $25,60 \%$ & $33,70 \%$ & $6,30 \%$ & $100 \%$ \\
\hline Slovakia & $34,00 \%$ & $13,10 \%$ & & $7,90 \%$ & $100 \%$ \\
\hline
\end{tabular}

Fuente: Alaminos, Penalva, Perea (2017) a partir de los datos del Eurobarómetro 364 (European Commission, Brussels, 2012)

En la tabla 3 podemos ver los resultados para cada país de esta pregunta. Hay que recordar que las preguntas que consideran hipótesis muestran por lo general un porcentaje elevado de no respuesta (Alaminos, 1994). Estos porcentajes son especialmente elevados en el caso de Austria, Alemania y Estonia, entre el 20 y el 25\%.

El análisis consiste en cruzar las respuestas a esta pregunta sobre preferencias electorales con los cuatro tipos expuestos más arriba (producto de una operacionalización sobre dos preguntas) sobre las concepciones acerca del derecho de sufragio en casos de movilidad intraeuropea. En conjunto, todas las preguntas tienen una opción de respuesta dicotómica. En todos los casos se 
ubica al entrevistado en una situación hipotética. Así, para preguntar sobre la adquisición o pérdida del derecho a voto (de donde se han obtenido los tipos de concepción de la ciudadanía) se proponen estas dos preguntas: “Consideremos la situación en la que un ciudadano de la Unión Europea vive en otro país de la Unión Europea" (a. debería conservar el derecho a voto en el país de origen? y b. Debería adquirir el derecho al voto en el país de destino?). De idéntico modo, en la otra variable se procede para preguntar sobre las preferencias de candidato. En el caso de la adquisición o pérdida del derecho al voto en elecciones nacionales las opciones son "sí" o "no", y en la preferencia por candidatos, la alternativa es entre candidatos del país de origen o candidatos del país de residencia.

Tabla 3. Preferencias de voto a candidatos según lugar de procedencia por país de la UE

\begin{tabular}{|c|c|c|c|c|}
\hline & $\begin{array}{l}\text { Candidatos } \\
\text { de su país de } \\
\text { residencia }\end{array}$ & $\begin{array}{l}\text { Candidatos } \\
\text { de su país } \\
\text { de origen }\end{array}$ & No sabe & Total \\
\hline Austria & $42,60 \%$ & $32,50 \%$ & $24,90 \%$ & $100 \%$ \\
\hline Belgium & $53,00 \%$ & $37,20 \%$ & $9,80 \%$ & $100 \%$ \\
\hline Bulgaria & $33,20 \%$ & $60,60 \%$ & $6,20 \%$ & $100 \%$ \\
\hline Cyprus (Republic) & $39,10 \%$ & $54,90 \%$ & $5,90 \%$ & $100 \%$ \\
\hline Czech Republic & $48,60 \%$ & $39,80 \%$ & $11,70 \%$ & $100 \%$ \\
\hline Germany & $49,20 \%$ & $28,70 \%$ & $22,00 \%$ & $100 \%$ \\
\hline Denmark & $36,20 \%$ & $52,10 \%$ & $11,70 \%$ & $100 \%$ \\
\hline Estonia & $31,50 \%$ & $48,80 \%$ & $19,70 \%$ & $100 \%$ \\
\hline Spain & $56,40 \%$ & $38,30 \%$ & $5,30 \%$ & $100 \%$ \\
\hline Finland & $36,70 \%$ & $57,90 \%$ & $5,40 \%$ & $100 \%$ \\
\hline France & $47,30 \%$ & $47,40 \%$ & $5,30 \%$ & $100 \%$ \\
\hline United Kingdom & $54,80 \%$ & $38,60 \%$ & $6,60 \%$ & $100 \%$ \\
\hline Greece & $47,20 \%$ & $47,90 \%$ & $4,90 \%$ & $100 \%$ \\
\hline Hungary & $52,10 \%$ & $39,00 \%$ & $8,80 \%$ & $100 \%$ \\
\hline Ireland & $54,40 \%$ & $39,90 \%$ & $5,70 \%$ & $100 \%$ \\
\hline Italy & $57,60 \%$ & $34,50 \%$ & $7,90 \%$ & $100 \%$ \\
\hline Lithuania & $25,30 \%$ & $64,80 \%$ & $9,90 \%$ & $100 \%$ \\
\hline Luxembourg & $51,90 \%$ & $40,00 \%$ & $8,10 \%$ & $100 \%$ \\
\hline Latvia & $36,10 \%$ & $52,50 \%$ & $11,40 \%$ & $100 \%$ \\
\hline Malta & $51,50 \%$ & $42,60 \%$ & $5,90 \%$ & $100 \%$ \\
\hline The Netherlands & $46,70 \%$ & $41,30 \%$ & $12,00 \%$ & $100 \%$ \\
\hline Poland & $35,00 \%$ & $55,00 \%$ & $10,00 \%$ & $100 \%$ \\
\hline Portugal & $39,30 \%$ & $57,40 \%$ & $3,30 \%$ & $100 \%$ \\
\hline Romania & $28,80 \%$ & $66,10 \%$ & $5,10 \%$ & $100 \%$ \\
\hline Sweden & $41,70 \%$ & $48,00 \%$ & $10,20 \%$ & $100 \%$ \\
\hline Slovenia & $42,10 \%$ & $48,50 \%$ & $9,50 \%$ & $100 \%$ \\
\hline Slovakia & $44,50 \%$ & $47,90 \%$ & $7,60 \%$ & $100 \%$ \\
\hline
\end{tabular}

Fuente: elaboración propia a partir de los datos del Eurobarómetro 364 (European Commission, Brussels, 2012) 
La hipótesis principal es que los diferentes tipos de orientaciones en la opinión pública con respecto a su concepción de ciudadanía (en términos de derecho de sufragio en casos de movilidad) está asociada a las preferencias en las elecciones europeas por un tipo de candidato $\mathrm{u}$ otro, caracterizado éste según el país de origen (ya sea connacional o del país de destino). De esta manera, se derivan las siguientes hipótesis:

- El signo de la asociación entre la orientación nacional (no se debería adquirir el derecho al voto en el país de destino) y la preferencia de voto del candidato de origen es positivo. Si una persona cree que los migrantes han de conservar únicamente el voto en el país de origen, en el caso de que ella misma emigrara a otro país (donde, a pesar de su opinión contraria tendría derecho a sufragio) votaría a sus connacionales. Esta coherencia expresa una especie sentimiento nacional que orienta tanto la concepción de ciudadanía basada en el origen y no como producto de la convivencia en otra comunidad política diferente a la de su propia nacionalidad; como su aprecio o valoración positiva hacia los condidatos compatriotas.

- El signo de la asociación entre la orientación nacional y la preferencia del candidato de destino es negativo. Sería la relación opuesta a la expuesta en la anterior hipótesis.

- El signo de la asociación entre la orientación estatal y la preferencia del candidato de destino es positiva. Para esta relación, la ciudadanía y el derecho a voto debe caminar hacia donde va el migrante, incluyendo la desconexión política con el país de origen. En términos de identidad política, la elección por parte de un individuo del candidato del país de destino es coherente con la concepción Estado orientado; por tanto, muestra una predisposición a entrar a formar parte de una ciudadanía nueva.

- La asociación entre la orientación cosmopolita y la preferencia del candidato de destino es positiva. Las actitudes serían muy similares a la orientación estatal. Aunque el votante no pierde su derecho a votar en el país de origen, considera importante su integración en el nuevo contexto (votar a candidatos del país de destino) por encima de los sentimientos nacionalistas (voto a candidatos connacionales).

\section{RESULTADOS}

En la tabla 4 podemos observar los resultados de la prueba de hipótesis realizada a partir del cruce entre las dos variables consideradas (concepción de ciudadanía y preferencia de candidatos). A pesar del considerable nivel de la no 
respuesta, las diferentes direcciones de los coeficientes para todos los países considerados permiten confirmar la relación de asociación. El considerable número de casillas con correlaciones significativas confirman las hipótesis.

Gráfico 1. Representación gráfica de la hipótesis

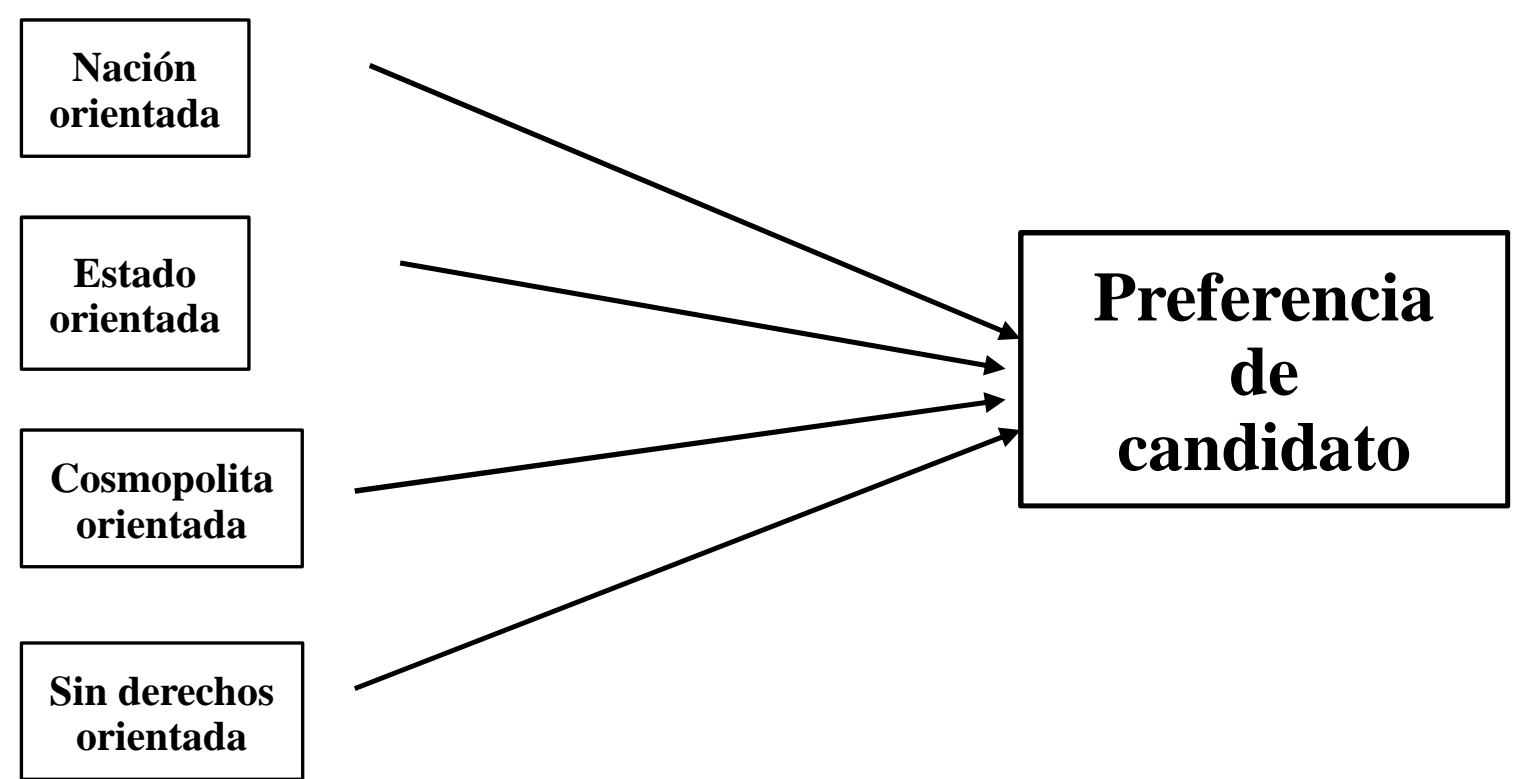

Tabla 4. Preferencia sobre el origen del candidato según tipo de orientación sobre el sufragio en 2012

\begin{tabular}{|l|l|c|c|c|c|}
\hline $\begin{array}{l}\text { Preferencia por candidato del país de destino } \\
(1) \text { o de origen (0) }\end{array}$ & $\begin{array}{c}\text { Nación } \\
\text { orientado }\end{array}$ & $\begin{array}{c}\text { Estado } \\
\text { orientado }\end{array}$ & $\begin{array}{c}\text { Cosmopolita } \\
\text { orientado }\end{array}$ & $\begin{array}{c}\text { Sin } \\
\text { derechos } \\
\text { orientado }\end{array}$ \\
\hline \multirow{4}{*}{ Austria } & Correlación de Pearson &,$- 192^{* *}$ &, $177^{* *}$ &, 028 &,- 010 \\
\cline { 2 - 6 } & Sig. (unilateral) &, 000 &, 000 &, 220 &, 392 \\
\cline { 2 - 6 } & $\mathrm{N}$ & 753 & 753 & 753 & 753 \\
\hline \multirow{4}{*}{ Belgium } & Correlación de Pearson &,$- 141^{* *}$ &, 009 &, $139^{* *}$ &,$- 056^{*}$ \\
\cline { 2 - 6 } & Sig. (unilateral) &, 000 &, 397 &, 000 &, 048 \\
\cline { 2 - 6 } & $\mathrm{N}$ & 903 & 903 & 903 & 903 \\
\hline \multirow{5}{*}{ Bulgaria } & Correlación de Pearson &,$- 150^{* *}$ &, $228^{* *}$ &,- 030 &, 027 \\
\cline { 2 - 6 } & Sig. (unilateral) &, 000 &, 000 &, 178 &, 207 \\
\cline { 2 - 6 } & $\mathrm{N}$ & 947 & 947 & 947 & 947 \\
\hline & Correlación de Pearson &,$- 249^{* * *}$ &, $111^{* *}$ &, $128^{* *}$ &,- 029 \\
\cline { 2 - 6 } & Sig. (unilateral) &, 000 &, 008 &, 003 &, 263 \\
\cline { 2 - 6 } & $\mathrm{N}$ & 476 & 476 & 476 & 476 \\
\hline \multirow{3}{*}{ Czech Republic } & Correlación de Pearson &,$- 125^{* *}$ &, 001 &, $128^{* *}$ &,- 048 \\
\cline { 2 - 6 } & Sig. (unilateral) &, 000 &, 491 &, 000 &, 079 \\
\cline { 2 - 6 } & $\mathrm{N}$ & 883 & 883 & 883 & 883 \\
\hline \multirow{2}{*}{ Germany } & Correlación de Pearson &,$- 204^{* *}$ &, $105^{* *}$ &, $075^{*}$ &,- 034 \\
\cline { 2 - 6 } & Sig. (unilateral) &, 000 &, 002 &, 019 &, 169 \\
\cline { 2 - 6 } & $\mathrm{N}$ & 780 & 780 & 780 & 780 \\
\hline
\end{tabular}


Si usted fuera migrante. Las preferencias de candidato como hipótesis

\begin{tabular}{|c|c|c|c|c|c|}
\hline \multirow[t]{3}{*}{ Denmark } & Correlación de Pearson &,$- 288^{* *}$ & $316^{* *}$ &, $077^{*}$ &,$- 060^{*}$ \\
\hline & Sig. (unilateral) & ,000 &, 000 & ,011 &, 036 \\
\hline & $\mathrm{N}$ & 891 & 891 & 891 & 891 \\
\hline \multirow[t]{3}{*}{ Estonia } & Correlación de Pearson &,$- 201^{* *}$ &, $096^{* *}$ & $101^{* *}$ & ,035 \\
\hline & Sig. (unilateral) & ,000 & ,003 & ,002 & 161 \\
\hline & $\mathrm{N}$ & 805 & 805 & 805 & 805 \\
\hline \multirow[t]{3}{*}{ Spain } & Correlación de Pearson &,$- 189^{* *}$ & $112^{* *}$ & $103^{* *}$ &,- 017 \\
\hline & Sig. (unilateral) &, 000 &, 000 & ,001 & ,303 \\
\hline & $\mathrm{N}$ & 947 & 947 & 947 & 947 \\
\hline \multirow[t]{3}{*}{ Finland } & Correlación de Pearson &,$- 265^{* *}$ &, $156^{* *}$ & $142^{* *}$ & ,045 \\
\hline & Sig. (unilateral) & ,000 &, 000 &, 000 & ,082 \\
\hline & $\mathrm{N}$ & 947 & 947 & 947 & 947 \\
\hline \multirow[t]{3}{*}{ France } & Correlación de Pearson &,$- 262^{* *}$ & ,202* & $134^{* *}$ &,$- 072^{*}$ \\
\hline & Sig. (unilateral) & ,000 & ,000 & ,000 &, 013 \\
\hline & $\mathrm{N}$ & 949 & 949 & 949 & 949 \\
\hline \multirow[t]{3}{*}{ United Kingdom } & Correlación de Pearson &,$- 163^{* *}$ &, $114^{* *}$ &, $062^{*}$ &,$- 082^{* *}$ \\
\hline & Sig. (unilateral) & ,000 &, 000 & ,028 &, 006 \\
\hline & $\mathrm{N}$ & 935 & 935 & 935 & 935 \\
\hline \multirow[t]{3}{*}{ Greece } & Correlación de Pearson &,$- 167^{* *}$ &, $058^{*}$ & $164^{* *}$ &,$- 082^{* *}$ \\
\hline & Sig. (unilateral) & ,000 &, 036 &, 000 & ,006 \\
\hline & $\mathrm{N}$ & 951 & 951 & 951 & 951 \\
\hline \multirow[t]{3}{*}{ Hungary } & Correlación de Pearson &,$- 126^{* *}$ & , $144^{* *}$ &,- 048 &, $125^{* *}$ \\
\hline & Sig. (unilateral) & ,000 &, 000 &, 073 & ,000 \\
\hline & $\mathrm{N}$ & 918 & 918 & 918 & 918 \\
\hline \multirow{3}{*}{ Ireland } & Correlación de Pearson &,$- 119^{* * *}$ & $134^{* *}$ &,- 011 &,$- 089^{* * *}$ \\
\hline & Sig. (unilateral) & ,000 & ,000 & 371 & ,003 \\
\hline & $\mathrm{N}$ & 943 & 943 & 943 & 943 \\
\hline \multirow[t]{3}{*}{ Italy } & Correlación de Pearson &,$- 208^{* *}$ &, $111^{* *}$ & $119^{* *}$ &,$- 063^{*}$ \\
\hline & Sig. (unilateral) & ,000 & , 000 & ,000 & ,027 \\
\hline & $\mathrm{N}$ & 921 & 921 & 921 & 921 \\
\hline \multirow[t]{3}{*}{ Lithuania } & Correlación de Pearson &,$- 149^{* *}$ & , $147^{* *}$ & ,041 & 034 \\
\hline & Sig. (unilateral) & ,000 & , 000 & 107 & 153 \\
\hline & $\mathrm{N}$ & 901 & 901 & 901 & 901 \\
\hline \multirow[t]{3}{*}{ Luxembourg } & Correlación de Pearson &,$- 099^{*}$ & , $151^{* *}$ & ,008 &,$- 172^{* * *}$ \\
\hline & Sig. (unilateral) & 017 & 001 & ,429 & ,000 \\
\hline & $\mathrm{N}$ & 464 & 464 & 464 & 464 \\
\hline \multirow[t]{3}{*}{ Latvia } & Correlación de Pearson &,$- 142^{* *}$ & $134^{* *}$ & ,002 &, $076^{*}$ \\
\hline & Sig. (unilateral) & , 000 & , 000 & ,475 & ,012 \\
\hline & $\mathrm{N}$ & 887 & 887 & 887 & 887 \\
\hline \multirow[t]{3}{*}{ Malta } & Correlación de Pearson &,$- 246^{* *}$ & $240^{* * *}$ & ,002 &,- 029 \\
\hline & Sig. (unilateral) &, 000 &, 000 & ,483 & ,265 \\
\hline & $\mathrm{N}$ & 479 & 479 & 479 & 479 \\
\hline \multirow[t]{3}{*}{ The Netherlands } & Correlación de Pearson &,$- 153^{* *}$ &, $088^{* *}$ &, $081^{* *}$ &,$- 103^{* * *}$ \\
\hline & Sig. (unilateral) & ,000 &, 004 & ,008 & ,001 \\
\hline & $\mathrm{N}$ & 883 & 883 & 883 & 883 \\
\hline \multirow[t]{3}{*}{ Poland } & Correlación de Pearson &,$- 085^{* *}$ & $186^{* *}$ &,$- 060^{*}$ & ,000 \\
\hline & Sig. (unilateral) & ,005 & ,000 & ,035 & ,494 \\
\hline & $\mathrm{N}$ & 900 & 900 & 900 & 900 \\
\hline \multirow[t]{2}{*}{ Portugal } & Correlación de Pearson &,$- 142^{* *}$ & $136^{* *}$ & ,021 &,- 012 \\
\hline & Sig. (unilateral) & 000 & ,000 & 253 & ,353 \\
\hline
\end{tabular}




\begin{tabular}{|c|c|c|c|c|c|}
\hline & $\mathrm{N}$ & 967 & 967 & 967 & 967 \\
\hline \multirow[t]{3}{*}{ Romania } & Correlación de Pearson &,- 007 &, 001 &,- 044 &, $075^{*}$ \\
\hline & Sig. (unilateral) & 410 & 490 & 086 &, 010 \\
\hline & $\mathrm{N}$ & 956 & 956 & 956 & 956 \\
\hline \multirow[t]{3}{*}{ Sweden } & Correlación de Pearson &,$- 301^{* *}$ & $183^{* *}$ & $180^{* * *}$ &,- 036 \\
\hline & Sig. (unilateral) & ,000 &, 000 &, 000 &, 143 \\
\hline & $\mathrm{N}$ & 898 & 898 & 898 & 898 \\
\hline \multirow[t]{3}{*}{ Slovenia } & Correlación de Pearson &,$- 191^{* *}$ & $211^{* *}$ & 049 &,- 017 \\
\hline & Sig. (unilateral) & ,000 & ,000 & 071 & ,303 \\
\hline & $\mathrm{N}$ & 906 & 906 & 906 & 906 \\
\hline \multirow[t]{3}{*}{ Slovakia } & Correlación de Pearson &,- 022 &, $056^{*}$ &,- 025 &,- 028 \\
\hline & Sig. (unilateral) & ,250 & ,045 & ,220 & ,202 \\
\hline & $\mathrm{N}$ & 924 & 924 & 924 & 924 \\
\hline
\end{tabular}

Fuente: elaboración propia con datos del Flash Eurobarómetro 364 de 2012 (European Commission, Brussels, 2012)

Al evaluar la relación entre la preferencia por los candidatos del país de origen o de destino con los tipos considerados puede apreciarse la consistencia de las actitudes y su transversalidad internacional. Observando la tabla en vertical se puede apreciar la dirección de las relaciones planteadas en las hipótesis para todos los países, detectándose a) una proximidad entre las concepciones de la ciudadanía orientada nacionalmente y sobre la pérdida de ciudadanía tras la emigración. Ambas se asocian negativamente con el voto a candidatos del país de destino (signos negativos en las columnas 1 y 4); y, de la misma manera, b) se aprecia la proximidad entre la concepción de ciudadanía orientada estatalmente y el derecho a la ciudadanía en ambos países tras la migración. Ambas se asocian positivamente (signos positivos en las columnas 2 y 3) con el voto a candidatos del país de destino.

Este incremento en la opción por el candidato de país de origen refleja muy probablemente un crecimiento, experimentado en los últimos años, en la orientación nacionalista. Así, considerando la tipología desarrollada sobre la base del derecho al voto se aprecia una consistencia elevada entre la orientación nacional y la preferencia por el candidato del país de origen. Esta consistencia se ve reforzada con la presencia del mismo signo (positivo) en la correlación entre ciudadanía sin derechos-orientada y preferencia por los candidatos connacionales.

Por otro lado, observando la tabla con una lectura horizontal vemos el grado de vertebración de la opinión pública, país a país, con respecto a la relación considerada. Las correlaciones son significativas en las cuatro columnas para países como The Nederlands, Denmark, Greece, France, Italy and United Kingdom. Países en los cuales va avanzando de forma notable el populismo nacionalista con los partidos de corte autoritario y xenófobo de Holanda (Partij 
voor de Vrijheid, PVV), Denmark (Dansk Folkeparti), Grecia (Amanecer Dorado/Golden Dawn), France (Front National), United Kingdom (United Kingdom Independence Party, UKIP) y en Italy (Lega Nord). En un grado menor, con tres coeficientes significativos, tenemos un gran grupo de países: Belgium, Cyprus, Germany, Estonia, Finland, Hungary, Ireland, Luxembourg, Latvia, Poland, Spain, Sweden; muy heterogeneo y, por último, un grupo donde están presentes la mayor parte de los países de la Europa Oriental, países con unas especificidades en su cultura política y sus sociedades políticas producto de los años de régimen comunista que son: con dos coeficientes significativos Austria, Bulgaria, Czech Republic, Lithuania, Malta, Portugal y Slovenia; o con únicamente uno Slovaquia y Rumania.

\section{DISCUSIÓN Y CONCLUSIONES}

Los resultados de este estudio transnacional acerca del estado de opinión de las sociedades políticas europeas en cuanto a sus preferencias electorales sobre la nacionalidad de los candidatos en un contexto de interculturalidad y sus concepciones acerca de la ciudadanía (utilizando como medición indirecta su opinión sobre en qué lugares se debe conservar o transferir el derecho al sufragio); permiten explorar las relaciones entre ciudadanía y nacionalidad que las sociedades perciben, en un contexto de intensificación de la movilidad intraeuropea. Un elemento clave expuesto en este trabajo es la impresión de que la opción por el candidato de país de origen refleja muy probablemente un crecimiento, experimentado en los últimos años en Europa, en la orientación nacionalista. El análisis de este estudio exploratorio proporciona diferentes grupos de países, cuyo criterio de clasificación ha sido el grado de coherencia entre su concepción de ciudadanía y su diferente identificación con candidatos connacionales o del país de residencia. Esta clasificación permite la formulación de nuevas hipótesis, y reflexionar sobre el papel que diferentes factores estructurales (cultura política, historia, estructura social y acervo jurídico e institucional) y coyunturales (económicos, fundamentalmente, pero también los relativos al estado de las relaciones internacionales) o de otros ámbitos de opinión (como son las actitudes hacia la Unión Europea), juegan a la hora de explicar los diferentes grados de consolidación de las estructuras de opinión sobre estos asuntos relacionados con la definición pública de la ciudadanía. Investigaciones futuras de mayor entidad (sobre nuevos indicadores y mayor amplitud muestral y temporal) permitiría realizar prospecciones sobre qué ocurrirá en un futuro no muy lejano en la Unión Europea; un futuro que puede arrastrar un contexto como el actual y sus efectos. Un contexto de crisis de la idea de Europa como proyecto político e intercultural, y de ausencia de una política común migratoria. 
Los proyectos políticos supranacionales como es el de la Unión Europea contienen un ligero cambio en la definición de los conceptos de soberanía como fuente de autoridad legítima dentro del Estado, y de ciudadanía como marco jurídico compartido por los individuos que forman una comunidad política. Por un lado, los estados miembros ceden parte de su soberanía a una entidad de orden superior como es la Unión y establecen canales de participación política en una realidad nueva marcada por la libre circulación de las personas; y, por otro, el concepto de ciudadanía también se matiza, incluyendo las personas que cambian de residencia en un contexto de movilidad intraeuropea. Es por ello que se ha ampliado el derecho al sufragio para ciertos procesos electorales (los locales y europeos), permitiendo la participación política (como candidatos o como electores) a todos los ciudadanos de la UE que son residentes en un país diferente al de origen.

Se trata, por tanto, de un proyecto institucional cuyo desarrollo no ha supuesto oposición, desde el punto de vista de la opinión pública (o de las opiniones públicas) de las sociedades europeas. En un contexto de desarrollo económico y de estabilidad política y social las sociedades europeas han participado y admitido sin apenas reservas la progresiva ampliación de la Unión (incorporando a buen número de países del Este europeo) y una extensión de los derechos de sufragio. Sin embargo, lo que está ocurriendo en los últimos años en Europa es una crisis económica y social que ha tenido sus efectos sobre los climas políticos nacionales y sobre la misma Unión Europea como proyecto político. Los datos de opinión pública que se presentan aquí muestran lo que puede ser un síntoma del cambio de tendencia en lo que se refiere a los sentimientos de identificación política con Europa. Frente al supranacionalismo, acorde con la creciente globalización cultural y económica en el ámbito internacional, se va situando el nacionalismo; y no sólo en el espacio europeo; un repliegue hacia las políticas nacionales soberanas (sin imposiciones foráneas) y un nacionalismo ideológico que lo sustenta. Se observan también, unas propuestas políticas con planteamientos dirigidos, en el ámbito económico, hacia el proteccionismo de la economía nacional y, en el ámbito de las políticas públicas, hacia la restricción de las ayudas y servicios sociales a los no nacionales. Si bien este estudio se centra en la movilidad intraeuropea, no deja de aparecer como referencia más o menos directa la creciente urgencia migratoria y humanitaria provocada por los conflictos bélicos pegados a la Unión Europea. Es un foco de tensión que en otros momentos históricos, con ausencia de crisis económica y social en Europa, no ha tenido tanta repercusión en el crecimiento de nacionalismos.

Por otro lado, si bien las opiniones sobre la ubicación del derecho de voto nos da pistas sobre las concepciones sociales y culturales que relacionan ciudadadanía y nacionalidad, no es menos cierto que este derecho sólo es una de las formas de participación política convencional. La evolución de este derecho político ha sido uno de los principales elementos constitutivos a partir 
de los cuales se han observado las transformaciones de los sistemas políticos occidentales desde la modernidad; derechos que han ido extendiéndose progresivamente conforme los sistemas políticos se han dio haciendo más abiertos e inclusivos sobre los individuos que residen en el territorio de un Estado. Los principios de representación y de soberanía aparecen ligados jurídica e ideológicamente para desarrollar los derechos de los individuos que forman una sociedad política. Así, el derecho al voto ha ido incorporando más individuos; desde la negación del sufragio del absolutismo hasta el derecho a votar por parte de las personas inmigrantes (ciudadanía transnacional), pasando por el sufragio censitario y, más adelante, por el sufragio universal, consecuencia este último de la asunción del principio democrático de igualdad. Una igualdad jurídica que la cultura política europea (o europeísta) ha intentado conjugar con la igualdad de oportunidades y la igualdad social; vigilando y actuando para que las brechas de desigualdad no se acrecentaran.

Se da la circunstancia de que las actuales fuerzas centrífugas, marcadas por el nacionalismo con respecto al proyecto europeo (y que este estudio detecta en las opiniones públicas de las sociedades europeas), van asociadas a procesos de centralización interna y reducción de derechos civiles y sociales, así como de negación de los derechos de las minorías. En este sentido, las decisiones de la Unión Europea deberían constituir una contrafuerza centrípeta a estos movimientos autoritarios y sus sustentos ideológicos. Se habría de incrementar la apuesta democrática. Si el déficit democrático de las instituciones europeas ha sido evidente desde sus inicios, déficit cuyas raíces están en el notable desequilibrio entre sus políticas económicas y sus políticas sociales; el momento actual debería ser el de cambiar sus políticas económicas de austeridad por unas más expansivas. Políticas que condujeran a un incremento del gasto público en servicios sociales para detener la polarización social y que tuvieran una visibilidad considerable de cara a las opiniones públicas de los diferentes estados miembros.

\section{REFERENCIAS BIBLIOGRÁFICAS}

Alaminos, A. (1996). Teoría y práctica de la encuesta. Madrid: CEDEAL.

Alaminos, A. (2002). "Maneras de vivir la sociedad". En: José Félix Tezanos

(ed.), Europa en Clase, Status y poder en las sociedades emergentes. Madrid: Editorial Sistema.

Alaminos, A., Penalva, C., Perea, N. (2017), La opinión pública europea y el derecho al voto de los migrantes, Sistema (en revisión)

Alter, P. (1994). Nationalism. London: Edward Arnold.

Arce Jiménez, C. (2012). Los derechos políticos de los residentes extranjeros: la ciudadanía inclusiva. Sevilla: Defensor del Pueblo Andaluz. 
Basch, L., Glick Schiller N., y Szanton-Blanc, C. (1994). Nations Unbound: Transnational Projects, Postcolonial Predicaments and Deterritorialized NationState. Amsterdam: Gordon \& Breach.

Bird, K., Saalfeld T. and Wüst, A.M. (2011). “The political representation of inmigrants and minorities. Voters, parties and parliaments in liberal democracies". London: Routledge.

Boli, J. y Thomas, G.M. (1999). "Chapter one INGOs and the Organization of World Culture", Constructing world culture: International nongovernmental organizations since 1875, , Stanford, Calif: Stanford University Press.

Brown, G. W. y Held, D. (2010). The cosmopolitanism reader. Cambridge: Polity Press.

Castles, S. (2003). “Jerarquías de ciudadanía en el marco del orden global”. Anales de la Cátedra Francisco Suárez, 37 (Ciudadanía e inmigración).

Estévez, A. (2016). “¿Derechos humanos o ciudadanía universal? Aproximación al debate de derechos en la migración". Revista Mexicana de Sociología, vol. 78 , no. 1 .

European Commission, Brussels (2014): Flash Eurobarometer 364 (Electoral Rights). TNS Political \& Social [producer]. GESIS Data Archive, Cologne. ZA5883 Data file Version 1.0.0, doi:10.4232/1.11931

Glick Schiller, N. y Fouron, G.E. (1999). “Terrains of Blood and Nation: Haitian Transnational Social Fields".Ethnic and Racial Studies, vol. 22, no. 2.

Glick Schiller, N., Basch, L. y Szanton-Blanc, C. (1992). "Towards a Definition of Transnationalism. Introductory Remarks and Research Questions". En: Glick Schiller, N., Basch, L. y Szanton-Blanc, C. (Eds.), Towards a Transnational Perspective on Migration: Race, Class, Ethnicity and Nationalism Reconsidered.New York: New York Academy of Sciences.

Guarnizo, L.E., Portes, A. y Haller, W. (2003). “Assimilation and Transnationalism: Determinants of Transnational Political Action among Contemporary Migrants". American Journal of Sociology, vol. 108, no 6.

Keck, M.E. y Sikkink, K. (1998). Activists beyond Borders: Advocacy Networks in International Politics. Ithaca, N.Y.: Cornell University Press.

Lafleur, J.M. (2012). “Transnacionalismo, diáspora y voto en el exterior". En: Lafleur, J.M. (Ed.), Diáspora y voto en el exterior. Barcelona: CIDOB.

León, B.O. (2015). “El derecho de sufragio como elemento estructural de la ciudadanía europea". Revista de derecho constitucional europeo, 4.

Levitt, P. y Glick Schiller, N. (2004). “Conceptualizing Simultaneity: A Transnational Social Field Perspective on Society1.". International Migration Review, vol. 38, no. 3.

Loughlin, J. (1994). "Representing Regions in Europe: The Committee of the Regions". Regional and Federal Studies, vol. 6, no 2.

Loughlin, J. (1996). Southern Europe Studies Guide.London: Bowker-Saur. 
Loughlin, J. (1999). La democracia regional y local en la Unión Europea.Bruselas: Comité de las Regiones.

Ludger Pries, L. (2001). "The disruption of social and geographic space mexican-us migration and the emergence of transnational social spaces". International Sociology, vol. 16, no 1.

Máiz, R. (2004). “Per Modum Unius: más allá de la dicotomía nacionalismo cívico/nacionalismo étnico". En: Gurrutxaga, A. El Presente del Estadonación. Bilbao: UPV.

Moldes, R. (2015). “De la "integración" a la "conectividad": expectativas laborales y formas de participación de la emigración cualificada". Arxius de Ciències Socials, No. 33.

Moraes, N. (2010). “Inclusión política, ciudadanía y territorio: algunos elementos para el debate sobre voto migrante". En: Sánchez, P. y Riella, A. (eds.), Globalización y perspectivas de la integración regional. Murcia: Universidad de Murcia.

Parlamento Europeo (2005). Informe sobre el Cuarto informe de la Comisión sobre ciudadanía de la Unión (1 de mayo de 2001 - 30 de abril de 2004), A60411/2005.

Parra, J.F. (2003). “Acercamiento al derecho de la migración y la ciudadanía transnacional. El caso de los emigrantes mexicanos y sus derechos políticos". América Latina Hoy, 33.

Portes, A. (1996). "Transnational Communities: Their Emergence and Significance in the Contemporary World System". En: Korzeniewicz, R.P. y Smith, W.C. (Eds.), Latin America in the World Economy. Westport, Conn: Greenwood Press.

Portes, A., Guarnizo, L.U. y Landolt, P. (1999) “The Study of Transnationalism: Pitfalls and Promise of an Emergent Research Field", Ethnic and Racial Studies, vol. 22, no. 2.

Smith, J., Chatfield, Ch. y Pagnucco, R. (Eds.) (1997). Transnational Social Movements and Global Politics: Solidarity beyond the State. Syracuse, N.Y.: Syracuse University Press.

Smith, M.P. y Guarnizo, L.E. (Eds.) (1998)." Transnationalism from Below". Comparative Urban and Community Research, vol. 6.

Tarrow, S. (1998). Power in Movement: Social Movements and Contentious Politics. Cambridge: Cambridge University Press.

Tortosa, J.M. (1999). “La Unión Europea: el difícil juego de tres nacionalismos”. En: Europa en la encrucijada. Zaragoza: Departamento de Educación y Cultura.

Velasco, J.C. (2009). "Transnacionalismo migratorio y ciudadanía en mutación". Claves de razón práctica, no 197.

Vertovec, S. (2003). “Transnational Migration: International Perspectives”. International Migration Review, vol. 37, no 3. 
Vertovec, S. (2007). "Super-diversity and its implications". Ethnic and racial studies, vol. 30, no 6.

ANTONIO FRANCISCO ALAMINOS CHICA es catedrático de Sociología Matemática en la Universidad de Alicante. Sus líneas de investigación son los métodos y técnicas de investigación social comparada, los procesos de socialización y aculturación y las dinámicas de cambio social y político.

CLEMENTE PENALVA VERDÚ es profesor titular en la Universidad de Alicante e investigador del Instituto Interuniversitario de Desarrollo Social y Paz. Sus principales líneas de investigación son capital social y movilización social, redes de apoyo mutuo y redes para la acción política, y la dimensión económica de la confianza social.

IGNACIA PEREA CRESPO es socióloga. Actualmente realiza el doctorado en la Universidad de Alicante donde ha colaborado en diversos proyectos de investigación con el Observatorio Europeo de Tendencias Sociales (OBETS) y el Instituto Interuniversitario de Desarrollo Social y Paz. Sus principales intereses de investigación son migraciones, multiculturalismo, aculturación y métodos de investigación social.

Recibido: 15/09/2016

Aceptado: 28/11/2016 